\title{
Isolation of Murine Primary Aortic Smooth Muscle Cells
}

Max Ole Hubert ${ }^{1}$, Juan Rodriguez-Vita ${ }^{1}$, Lena Wiedmann ${ }^{1}$ and Andreas Fischer ${ }^{1,2,3}$, *

1Division of Vascular Signaling and Cancer, German Cancer Research Center (DKFZ), Heidelberg,

Germany; ${ }^{2}$ European Center for Angioscience, Medical Faculty Mannheim, Heidelberg University, Mannheim, Germany; ${ }^{3}$ Medical Clinic I, Endocrinology and Clinical Chemistry, Heidelberg University Hospital, Heidelberg, Germany

*For correspondence: a.fischer@dkfz.de

[Abstract] Vascular smooth muscle cells (VSMCs) have been cultured for decades to study the role of these cells in cardiovascular disorders. The most common source of VSMCs is the rat aorta. Here we show the adaptation of this method to isolate and culture mouse aortic VSMCs. The advantage of this method is that there are many more transgenic mouse lines available compared to rats. The protocol consists of the isolation of the aorta, the liberation of vascular cells by the action of collagenase, culturing of VSCMs, and analyzing filamentous actin and alpha smooth muscle actin by fluorescence microscopy. VSCMs can be further used to study mechanisms underlying cardiovascular diseases.

\section{Graphic abstract:}

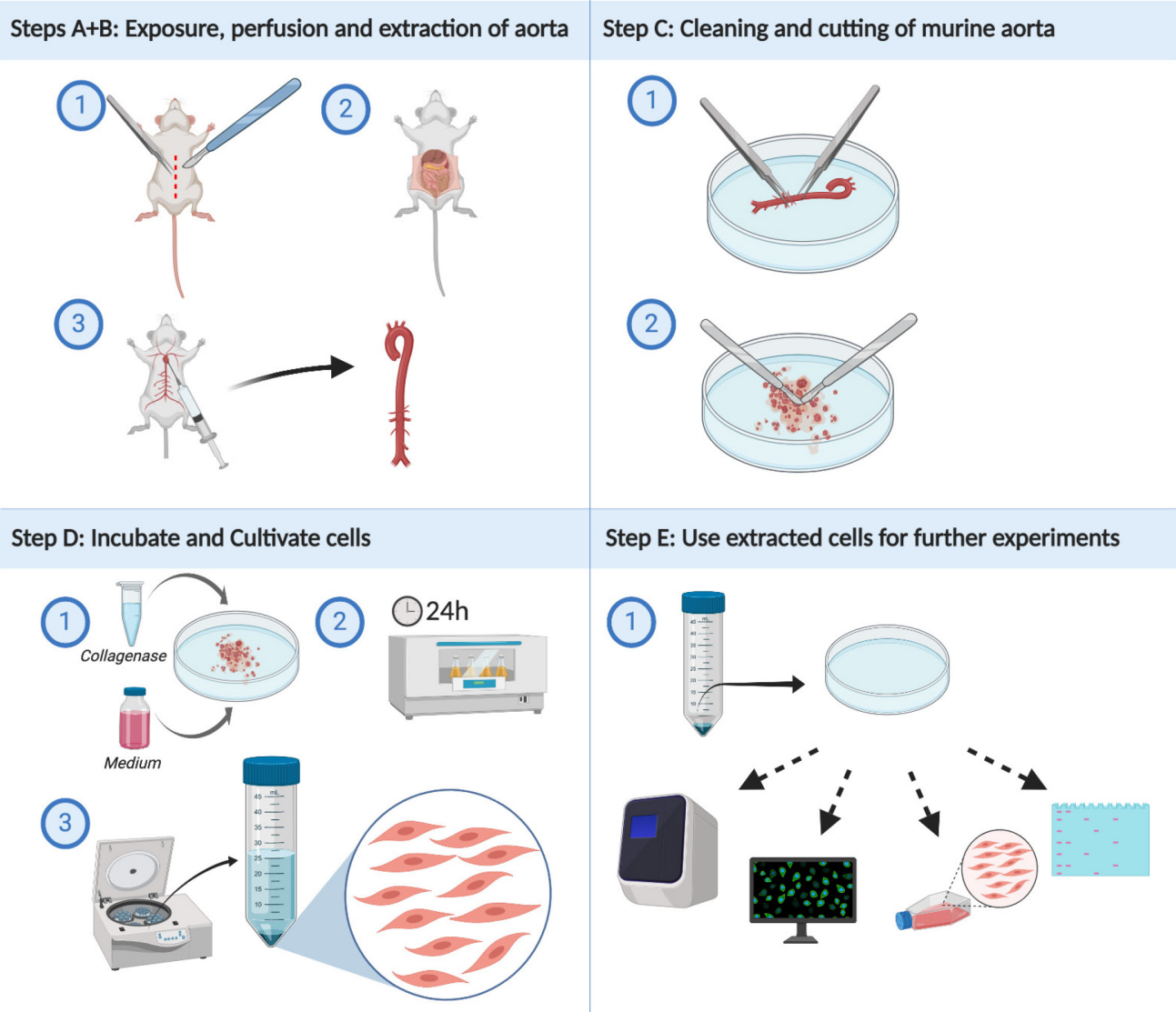

Figure 1. Working steps 
Keywords: Vascular smooth muscle cells, Murine aorta, Extraction, Isolation, Cultivation

[Background] Cells of the blood vessel wall (mural cells) are critically involved in the regulation of several disorders, from hypertension (Rodríguez-Vita et al., 2005a) and atherosclerosis (Rodríguez-Vita et al., 2008) to cancer (Wong et al., 2020) and more recently even COVID-19 (He et al., 2020). Whereas capillaries are mostly covered by pericytes, larger blood vessels contain VSCMs in the vessel wall. Mural cells control blood flow by vasoconstriction and vasodilation. As such, they are the target of several drugs which lower blood pressure (St. Paul et al., 2020). VSMCs stabilize blood vessels and are involved in restricting vessel permeability. Mural cells also limit angiogenesis and the presence of a tight VSMCs network is a feature of mature vessels (Hungerford and Little, 1999). VSMCs can also get activated and transdifferentiated into fibroblasts which contribute to tissue fibrosis (Rodríguez-Vita et al., 2005a and $2005 b)$. In summary, the unique role of VSCMs in the maintenance of vascular homeostasis and control of blood flow makes cultured VSMCs a valuable tool in studying cellular and molecular mechanisms of vascular biology.

Here we describe a method to culture VSMCs from mouse aortas. This method is an adaptation from a method employed in rats 15 years ago (Rodríguez-Vita et al., 2005b). It allows evaluating the specific role of VSMCs in vitro in pathological processes observed in vivo. The advantage of primary culture allows to study genetic manipulation in these cells by using aortas derived from transgenic mice. The high viral or liposomal transduction efficiency of these cells also allows to study the role of other genes for which no transgenic mice are available. There are a number of molecular analysis that can be performed with cultured VSMCs such as gene expression analysis, drug screening, cell migration, proliferation, or survival assays. For example, we employed this method recently to analyze the role of HTRA1 in VSMC maturation (Klose et al., 2019).

\section{Materials and Reagents}

1. Pipette tips $(10 \mu \mathrm{l}, 200 \mu \mathrm{l}, 1,000 \mu \mathrm{l})$ (Starlab, catalog numbers: S1111-3700, S1111-0706, S1111-6701)

2. $60 \mathrm{~mm}$ cell culture dish (Falcon, Corning, catalog number: 353 004)

3. $15 \mathrm{ml}$ conical tube (Corning, catalog number: 352096 )

4. $50 \mathrm{ml}$ conical tube (Corning, catalog number: 352070 )

5. Greiner CELLSTAR ${ }^{\circledR}$ multiwell culture plates 24 wells (TC treated with lid) (Merck, catalog number: M8812)

6. Thermo Scientific ${ }^{\mathrm{TM}}$ SuperFrost Plus ${ }^{\mathrm{TM}}$ Adhesion slides (Thermo Scientific Fisher, catalog number: 10149870)

7. Cover slips, circular, $24 \mathrm{~mm}$ (Th.Geyer, catalog number: 7629906)

8. Mouse

9. Ethanol $70 \%$ (VWR, catalog number: 20821.330)

10. FBS (Merck, Biochrom, catalog number: S 0615) 
11. DPBS, w/o calcium and magnesium (Thermo Fisher Scientific, catalog number: 14190169)

12. DMEM, low glucose, GlutaMAX, pyruvate (Thermo Fisher Scientific, catalog number: 21885108)

13. Collagenase, Typ II (Thermo Fisher Scientific, catalog number: 17101015)

14. Fungizone-Amphoterecin B (Thermo Fisher Scientific, catalog number: 15290018)

15. Penicillin-Streptomycin (Thermo Fisher Scientific, catalog number: 15140122)

16. Tween $^{\circledR} 20$ for molecular biology (AppliChem, catalog number: A4974)

17. Glycin $\geq 99 \%$, Blotting Grade (Carl Roth, catalog number: 0079.3)

18. ROTI ${ }^{\circledR}$ Histofix $4 \%$ (Carl Roth, catalog number: P087.3)

19. Triton ${ }^{\circledR}$ X-100 Molecular Biology grade (AppliChem, catalog number: A4975)

20. Alexa Fluor ${ }^{\mathrm{TM}} 488$ Phalloidin (Thermo Fisher Scientific, catalog number: A12379)

21. Invitrogen ${ }^{\mathrm{TM}}$ DAPI (4',6-Diamidino-2-Phenylindole, Dilactate) (Thermo Fisher Scientific, catalog number: D3571)

22. Fluorescence Mounting Medium (Agilent, catalog number: S3023)

23. Incubation-collagenase media (see Recipes)

24. Permeabilization buffer (see Recipes)

25. Blocking buffer (see Recipes)

\section{Equipment}

1. One Channel Pipette (Volume 1-10 $\mu \mathrm{l}$ ) (Eppendorf, catalog number: 3124000024)

2. One Channel Pipette Volume 10-100 $\mu$ l) (Eppendorf, catalog number: 3124000075)

3. One Channel Pipette (Volume 100-1,000 $\mu$ l) (Eppendorf, catalog number: 3124000121)

4. Hemacytometer; Neubauer counting chamber (BRAND, catalog number: 717805)

5. PIPET-Boy acu2 (Integra Biosciences, catalog number: 155000)

6. Forceps Shandon, 10 inches (Thermo Scientific Fisher, catalog number: 6306)

7. Spring Scissors fine, small blade, straight, $8 \mathrm{~cm}$ (Fine Science Tools, catalog number: 1500003)

8. Single-Use Scalpel, no.20 (Paragon, neoLab, catalog number: 1-1565)

9. Autoclave (VWR, Tuttnauer, catalog number: 481-0585)

10. Balance (KERN \& SOHN, catalog number: PBJ 4200-2M)

11. Centrifuge with swinging-bucket rotor and adaptors for $15 \mathrm{ml}$ and $50 \mathrm{ml}$ conical tubes (Eppendorf, model: 5810 , catalog number: 5810000320 )

12. Humidified cell culture incubator set to $37^{\circ} \mathrm{C}$ and $5 \% \mathrm{CO}_{2}$ (Thermo Fisher Scientific, Heracell ${ }^{\mathrm{TM}}$ 150CU, catalog number: 50116047)

13. Light microscope (Leica Microsystems, model: Leica DM IRB)

14. Safety cabinet (Thermo Fisher Scientific, model: Safe 2020, catalog number: 51026638)

15. Water bath (GFL, catalog number: 1012)

16. Zeiss Cell Observer Motorized Widefield Microscope (Zeiss) 


\section{Procedure}

\section{Timeline}

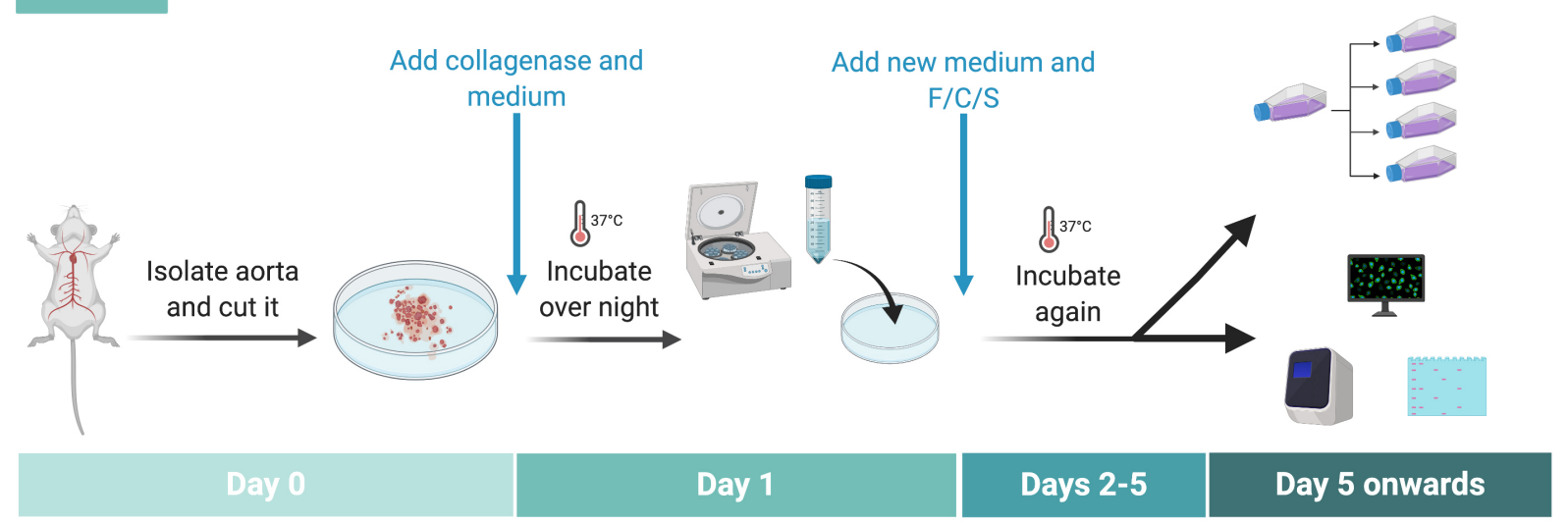

Figure 2. Timeline of the procedure

A. Preparing the mouse

1. Set up a surgery table with the above-described equipment.

2. Euthanize the mouse by cervical dislocation. Alternative methods may be used granted euthanasia is obtained.

3. Spray the surfaces you will work on with $70 \%$ ethanol. Spray the abdominal region of the mouse with ethanol.

Note: The ethanol does not only sanitize the mouse's abdominal region it also fixes its fur. This avoids having hair in the preparation field.

4. Place the mouse on a surgical board in a supine position with upper and lower extremities stretched outwards.

5. Use four fixation needles to secure the position of the animal's extremities.

B. Preparation and extraction of the murine aorta (see Figure 1 Steps $A+B$ )

1. Open the fur and skin layer of the mouse using forceps and scissors.

2. To do so incise in the median line of the mouse, roughly above the xiphoid and enlarge your initial incision into both directions up and down.

3. Leave the thoracic and abdominal cavity closed and dissect all skin layers exposing the peritoneum and thoracic region.

4. Now open up the abdominal cavity using forceps and scissors. Strongly lift the peritoneal layers to make sure you do not cut into the intestine.

Note: For a clean skin dissection as well as the opening of the thoracic and abdominal cavity, it is crucial to create sufficient tension on the tissue with the forceps to cleanly incise. Especially while opening up the abdominal cave you can hereby avoid to puncture the intestine and thereby contaminate your samples with intestinal bacteria. 
5. After that, open up the thoracic cavity by dissecting the diaphragm, be careful not to puncture the heart during dissection of the diaphragm.

6. Remove the lung. Grab lobe by lobe and excise with a scissor.

7. Remove the abdominal organs to gain sight onto the aorta. Initially do not use a scalpel or scissors for the removal of the abdominal organs. Take rough tissue instead and smoothly spread away the intestine and the other organs to both sides.

Note: Once the organs are located outside the peritoneal cavity, on both sides of the mouse, you can use a scalpel or scissors to strip away the organs from their connecting tissue and meso. Even if you lacerate one of the intestinal or other abdominal organs in this step you avoid contamination of your future samples because of the previous steps.

8. Cut both femoral arteries open to allow blood and solution to flow out during perfusion.

9. Cannulate left ventricle and perfuse with $10 \mathrm{ml}$ of ice cold PBS + P/S/F.

10. Now extract the murine aorta (see Video 1). In order to resect the aorta as clean as possible hold it with the forceps at the distal end and firmly lift it up. Then cut the underlying tissue which should be under tension at any time.

Note: Dissection is most difficult at the branches of the renal arteries and the passing point of the aorta through the diaphragm as the vessel is attached to its surrounding tissue quite firmly at those areas.

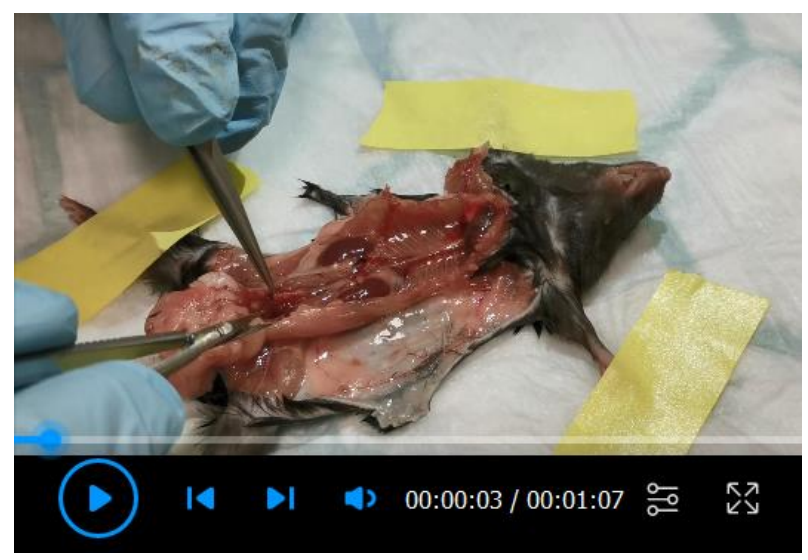

Video 1. Aorta extraction. (This video was made at German Research Cancer Center Heidelberg according to guidelines from the German Cancer Research Center and Heidelberg University on Animal Care and approved by the Animal Research Ethics Board of German Cancer Research Center Heidelberg under protocol \# DKFZ 244.)

C. Cleaning and Cutting of the murine aorta

1. Place the extracted aorta on a $60 \mathrm{~mm}$ dish (use the top of the dish so that the sidewalls do not disturb your preparation actions) (see Figure 1 step $\mathrm{C} 1$ ).

2. If possible use curved forceps and remove all the surrounding adipose and fibrous tissue and adventitia (see Video 2) (see Figure 3 for the final result). 
Note: The cleaning will be much easier in the thoracic part than in the abdominal of the aorta due to branch structure.

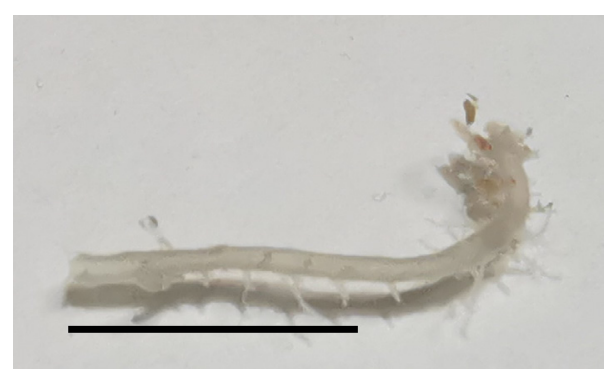

Figure 3. Clean aorta after adventitia removal. The scale bar is $1 \mathrm{~cm}$.

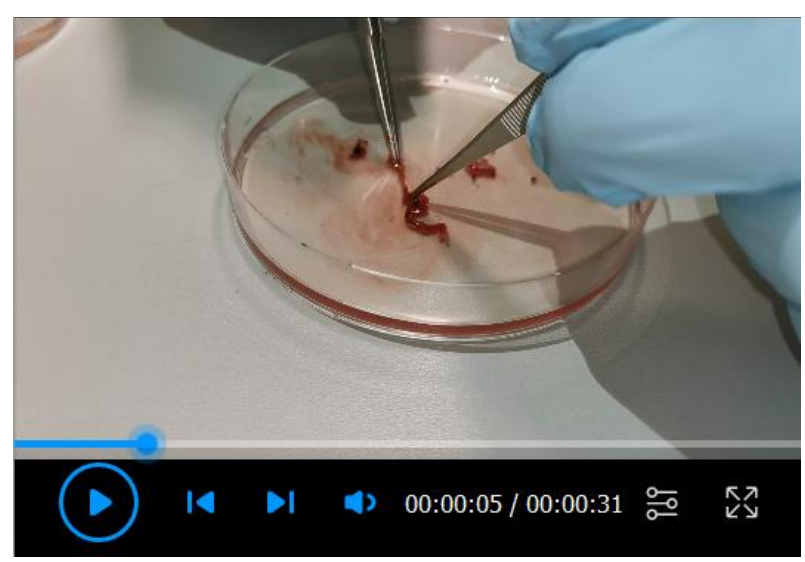

\section{Video 2. Cleaning}

3. Now place the cleaned aorta on a fresh $60 \mathrm{~mm}$ dish to cut it into small rings. We recommend using two scalpels for the cutting (see Video 3). Practice has shown that this method is superior to using one forceps and one scalpel regarding fineness of the cuttings (see Figure 1 step C2 and Figure 2).

4. Add $2 \mathrm{ml} \mathrm{DMEM+15 \%} \mathrm{FCS+P/S/F} \mathrm{(PenStrep,} \mathrm{Fungizone)} \mathrm{+} \mathrm{collagenase} \mathrm{(concentration} 10$ $\mathrm{mg} / \mathrm{ml}$, so in this case $20 \mathrm{mg}$ ) (see Figure 1 step D1 and Figure 2).

5. Incubate overnight at $37^{\circ} \mathrm{C}$ with $5 \% \mathrm{CO}_{2}$ (see Figure 1 step D2). 


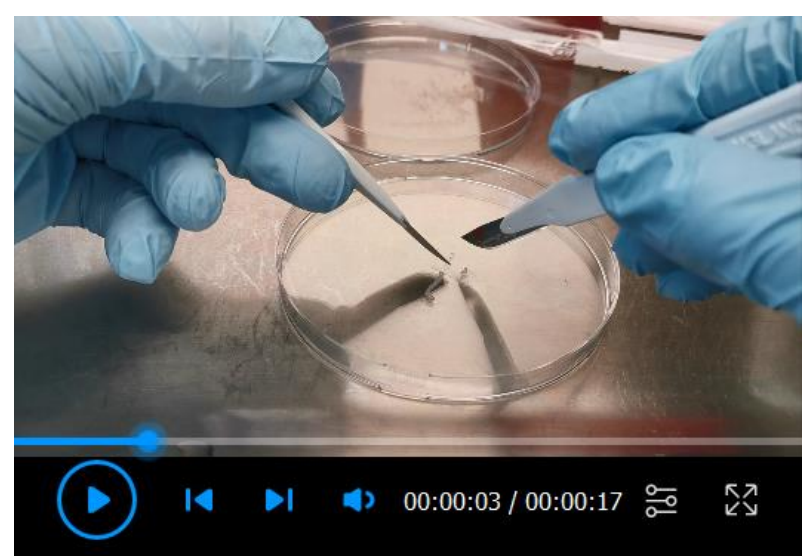

Video 3. Cutting

D. Cultivation of vascular smooth muscle cells

1. Next day: Pipette the medium containing the aortic tissue up and down and rinse the culture dish thoroughly. This helps to disrupt adhesive rings by shear forces.

2. Transfer the media with the sample tissue into a $15 \mathrm{ml}$ Falcon and centrifuge $5 \mathrm{~min}$ at $180 \times \mathrm{g}$ (see Figure 1 step D3).

3. Discard the supernatant and resuspend the pellet with DMEM+FCS+P/S/F and plate it in a new cell culture dish (see Figure 1 step E1).

Note: At this point, you might want to pool the cells from two to three murine aortas. The yield from one aorta should be enough for simple procedures such as qPCR or immunohistochemistry. However, we recommend pooling the extracted cells from at least two mice for more complex procedures that require a higher number of VSMCs. All pooled cells should be cultured in one dish together. To achieve the best results out of the following cultivation of the cells we recommend using a maximum dish size of $60 \mathrm{~mm}$ as the number of cells you will win by the extraction is not expected to be high enough for a $100 \mathrm{~mm}$ dish. Tissue culture dishes should be used to ensure adherence, extra coating of the dish is not necessary.

4. Check after 3-5 days (see Figure 2) if vascular smooth muscle cells adhere to the bottom of the dish (do not change the dish or media at this point) (see Figure 4).

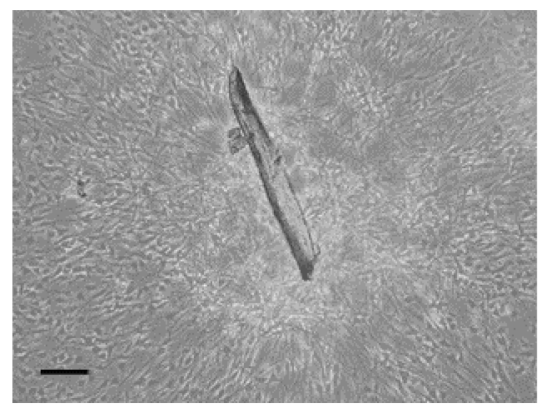

Figure 4. Cells after 4 days after isolation. It is visible how the cells start to detach from the aortic pieces. The scale bar is $100 \mu \mathrm{m}$. 
5. Change the medium (DMEM $+15 \%$ FCS) every 2 days and passage the cells only when confluence is achieved.

E. Quality control

In order to know whether the cells are in proper conditions, actin fibers can be stained. We will briefly describe a staining protocol for filamentous actin (F-actin) (see Figure 5).

1. Seed the obtained vascular smooth muscle cells on glass cover slips in a 24-well format in DMEM with $15 \%$ FCS.

2. 2-7 days later, fix the attached cells with Histofix (or $4 \%$ paraformaldehyde) for $10 \mathrm{~min}$ at room temperature.

3. Remove the Histofix by washing with PBS.

4. To permeabilize, incubate cells with permeabilization buffer (see Recipe 2) for $10 \mathrm{~min}$.

5. Wash with PBS.

6. To prevent unspecific binding of antibodies, block for 30 min with blocking buffer (see Recipe 3 ).

7. Incubate the cells with Alexa Fluor ${ }^{\mathrm{TM}} 488$ Phalloidin (1:200) in blocking buffer for $1 \mathrm{~h}$ at room temperature in the dark.

Note: From now on exposure to light should be reduced to the minimal possible extend to avoid photobleaching. Since most of the undesired cell types are present in the adventitia, the cells left in the media and intima, which is the part that is processed, will restrict to endothelial and smooth muscle cells (see Steps C1-C2). Endothelial cells need collagen coating to be able to adhere to the plate, considering that the aortas are incubated with collagenase, it would not be possible for the endothelial cells to adhere to the plate. Moreover, endothelial cells need a very special medium for their survival. It is extremely unlikely that they would be contaminating the culture after Step D5. However, if contamination would want to be evaluated cells could be stained with CD31 to detect endothelial cells. This single staining does not only function as a control of the cells' state but also proofs purity of the culture. As the aorta was cleaned from all surrounding tissue (see Steps C1-C2) only VSMCs and endothelial cells are expected to be cultured. The endothelial cells will not survive the culturing as they would require certain conditions so purity is ensured by the cleaning step.

8. Wash $3 \times$ with PBS.

9. Incubate with DAPI in PBS (final concentration $2 \mu \mathrm{M}$ ) for $10 \mathrm{~min}$ at room temperature.

10. Wash $3 \times$ with PBS.

11. Mount coverslips on slides with fluorescence mounting medium. Store until analysis at $4{ }^{\circ} \mathrm{C}$.

12. Analyze staining at a fluorescent microscope, e.g., Zeiss Cell Observer (see Figure 5). 


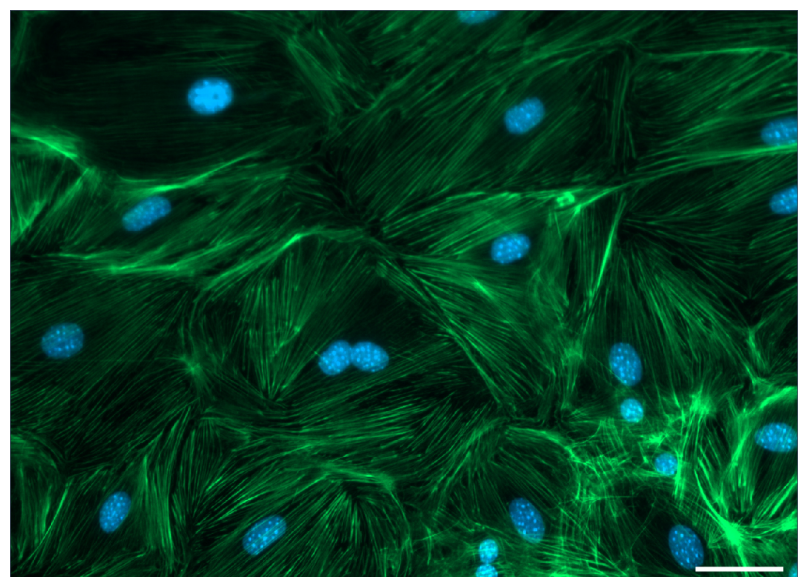

Figure 5. Vascular smooth muscle cells stained for F-Actin by Phalloidin (green) and DAPI (blue). Scale bar: $50 \mu \mathrm{m}$.

\section{$\underline{\text { Recipes }}$}

1. Incubation-collagenase media

Make sure to have a final concentration of $10 \mathrm{mg} / \mathrm{ml}$ of collagenase in the media in which you incubate the sample overnight.

Lower concentrations will impact your amount of cells profoundly.

2. Permeabilization buffer

PBS

$5 \%$ FBS

$0.01 \%$ Tween-20

$0.75 \%$ glycine

$0.1 \%$ Triton-X 100

3. Blocking buffer

PBS

$5 \%$ FBS

$0.01 \%$ Tween-20

$0.75 \%$ glycine

\section{Acknowledgments}

This work was supported by the Deutsche Forschungsgemeinschaft (SFB1366 no. 394046768 project C4 and project no. 419966437).

\section{Competing interests}

The authors declare that they have no conflict of interest. 


\section{Ethics}

All animal experiments had been approved by the responsible agencies. The animal experiments were conducted using the approval ID DKFZ244.

\section{$\underline{\text { References }}$}

1. He, L., Mäe, M. A., Sun, Y., Muhl, L., Nahar, K., Liébanas, E. V., Fagerlund, M. J., Oldner, A., Liu, J., Genové, G., et al. (2020). Pericyte-specific vascular expression of SARS-CoV-2 receptor ACE2 - implications for microvascular inflammation and hypercoagulopathy in COVID-19 patients. bioRxiv: 2020.2005.2011.088500.

2. Hungerford, J. E. and Little, C. D. (1999). Developmental biology of the vascular smooth muscle cell: building a multilayered vessel wall. $J$ Vasc Res 36(1): 2-27.

3. Klose, R., Prinz, A., Tetzlaff, F., Weis, E.-M., Moll, I., Rodriguez-Vita, J., Oka, C., Korff, T. and Fischer, A. (2019). Loss of the serine protease HTRA1 impairs smooth muscle cells maturation. Sci Rep 9(1): 18224.

4. St. Paul, A., Corbett, C. B., Okune, R. and Autieri, M. V. (2020). Angiotensin II, Hypercholesterolemia, and Vascular Smooth Muscle Cells: A Perfect Trio for Vascular Pathology. Int J Mol Sci 21(12).

5. Rodríguez-Vita, J., Ruiz-Ortega, M., Rupérez, M., Esteban, V., Sanchez-López, E., Plaza, J. J. and Egido, J. (2005b). Endothelin-1, via ETA receptor and independently of transforming growth factor- $\beta$, increases the connective tissue growth factor in vascular smooth muscle cells. Circulation Res 97(2): 125-134.

6. Rodríguez-Vita, J., Sanchez-Galán, E., Santamaria, B., Sánchez-López, E., Rodrigues-Díez, R., Blanco-Colio, L. M., Egido, J., Ortiz, A. and Ruiz-Ortega, M. (2008). Essential role of TGFbeta/Smad pathway on statin dependent vascular smooth muscle cell regulation. PLoS One 3(12): e3959.

7. Rodríguez-Vita, J., Sánchez-López, E., Esteban, V., Rupérez, M., Egido, J. and Ruiz-Ortega, M. (2005a). Angiotensin II activates the Smad pathway in vascular smooth muscle cells by a transforming growth factor-beta-independent mechanism. Circulation 111(19): 2509-2517.

8. Wong, P. P., Munoz-Felix, J. M., Hijazi, M., Kim, H., Robinson, S. D., De Luxan-Delgado, B., Rodriguez-Hernandez, I., Maiques, O., Meng, Y. M., Meng, Q. et al. (2020). Cancer Burden Is

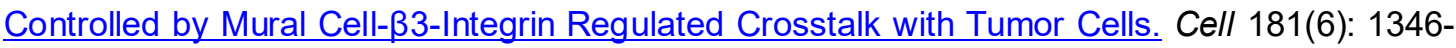
1363 e1321. 